\title{
Preparation and Characteristics of a Novel Sustained and Controlled Release Drug Delivery Device by Plasma Technique
}

\author{
Yukinori Yamauchi ${ }^{1}$, Masayuki Kuzuya ${ }^{2}$, Yasushi Sasai ${ }^{3}$, \\ Naoki Doi ${ }^{3}$, and Shin-ichi Kondo ${ }^{3}$ \\ ${ }^{1}$ Department of Pharmaceutical Physical Chemistry, College of \\ Pharmaceutical Sciences, Matsuyama University, 4-2 Bunkyo-cho, Matsuyama, \\ Ehime 790-8578, Japan \\ ${ }^{2}$ Department of Health \& Welfare, Chubu Gakuin University, 2-1, Kirigaoka, \\ Seki-shi, Gifu, 501-3993, Japan \\ ${ }^{3}$ Laboratory of Pharmaceutical Physical Chemistry, Gifu Pharmaceutical \\ University, 1-25-4 Daigaku-Nishi, Gifu 501-1196, Japan
}

Keywords: Oxygen plasma irradiation, drug delivery system, cellulose derivatives

\section{Introduction}

Increased complications and expense involved in marketing of new drug entities has focused greater attention on development of sustained and controlled release drug delivery systems [1]. The purpose of the systems is to maintain drug concentration in the blood or in target tissues at a desired value as long as possible. To achieve predictable and reproducible release rates, extended duration of activity for short half-life drugs, decreased toxicity, and reduction of required dose, optimized therapy and better patient compliance, a sustained-release (SR) formulation of various drugs is desirable [2].

Over the years, we have been working on the development of plasma-assisted preparation of multi-layered tablets applicable to oral drug delivery system (DDS) [3-5]. In previous papers, we reported the preparation of SR system by oxygen plasma-irradiation to the doublecompressed tablet having a mixture of plasmacrosslinkabe material, like polystyrene (PSt) or hydroxypropyl methylcellulose acetate succinate (HPMCAS), and plasma-degradable material, like polyoxymethylene (POM) or lactose, as a wall material $[6,7]$. When oxygen plasma was irradiated to the outermost layer of the tablet, plasma-degradable material could be selectively eliminated and simultaneously plasma-cross- linkable material undergoes the rapid crosslink reaction to result in the formation of the porous outer layer of the tablet. As a result, the drugs could be released slowly from the tablet through the resulting micropores.

The objective of this study was to develop once-daily sustained-release dosage forms, which releases drug at zero-order rate with accurate control in the gastrointestinal tract.

\section{Experimental}

\subsection{Materials}

HPMCAS (AS-LF, Fig.1) was kindly supplied by Shin-Etsu Chemical Co. (Tokyo, Japan). Lactose monohydrate (Fig.1) was purchased from Wako Pure Chemical Industry Co. (Tokyo, Japan). All other materials used were of analytical grade, and procured from commercial sources.

\subsection{Plasma-Irradiation and Tablet Preparation}

Tested powders or tablets were treated with radio frequency $(13.56 \mathrm{MHz})$ plasma.

The double-compressed tablets were obtained as follows: a flat-faced core diclofenac sodium tablet $(50 \mathrm{mg}, 10 \mathrm{~mm} \phi)$, as a model drug, was first prepared at a pressure of $40 \mathrm{~kg} / \mathrm{cm}^{2}$ for $10 \mathrm{~s}$, and then the core tablet was placed onto half of the prescribed amount of powdered mixture (50 $\mathrm{mg}$ ) as a wall material in a tablet die. After the 
rest of the powdered mixture $(50 \mathrm{mg}$ ) was placed on the core tablet, the whole was compressed at pressure of $200 \mathrm{~kg} / \mathrm{cm}^{2}$ for $30 \mathrm{~s}$. All flat-faced tablets were prepared using a hand press instrument in a tablet die (SE work press and SE dice, Okada seiko Co., Japan).
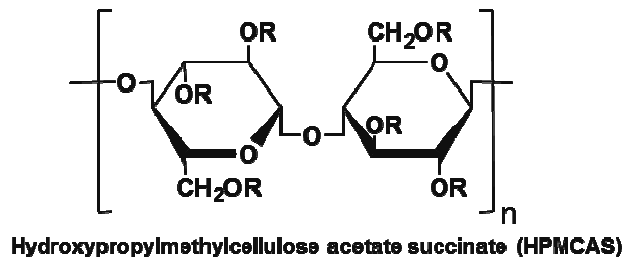

$\mathrm{R}=-\mathrm{H},-\mathrm{CH}_{3},-\mathrm{CH}_{2} \mathrm{CH}\left(\mathrm{CH}_{3}\right) \mathrm{OH},-\mathrm{COCH}_{3}$, or $-\mathrm{COCH}_{2} \mathrm{CH}_{2} \mathrm{COOH}$ (AS-LF: methoxyl 22\%, hydroxypropoxy $8 \%$, acetyl $8 \%$, succinoyl 15\%)

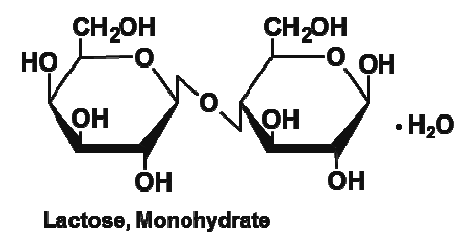

Fig. 1. Chemical structures of HPMCAS and Lactose monohydrate.

The apparatus and methods for plasmairradiation on both powder and tablet is essentially the same as reported earlier [3-7]. The plasma state was generated by use of capacitively coupled parallel-plate radio frequency discharges at $13.56 \mathrm{MHz}$ with supplied power of $50 \mathrm{~W}$. Flow volume $(50 \mathrm{~mL} / \mathrm{min})$ and pressure of argon or oxygen gas $(66.6 \mathrm{~Pa})$ for plasmolysis were controlled by changing evacuating speed. The sample tablets were placed on a glass-tripod in a reaction chamber (240 $\mathrm{mm}$ long, $150 \mathrm{~mm} \phi)$ to ensure homogeneous exposure to plasma gas.

Degradation rate of sample tablets induced by plasma-irradiation was carried out according to the method similar to the above, and determined by measurement of the weight loss at various stages of plasma duration.

\subsection{Scanning Electron Microscope (SEM)}

The microscopic changes in the surface of the plasma-irradiated powders and tablets were evaluated by SEM (S-3400N, Hitachi, Japan) with an accelerating voltage of $5 \mathrm{kV}$ and magnification of $\mathrm{x} 500-2,000$. Prior to the analyses, the samples were sputtered with a thin film of gold.

\subsection{In Vitro Release Studies}

The in vitro dissolution studies were carried out using a dissolution test apparatus with an autosampling apparatus (Toyama Sangyo Co., Osaka, Japan) at $100 \mathrm{rpm}$. The dissolution medium consisted of the $0.1 \mathrm{M}$ phosphate buffer $\mathrm{pH} 7.4(900 \mathrm{~mL})$, maintained at $37 \mathrm{C} \pm 0.5^{\circ} \mathrm{C}$. The drug release at different time intervals was measured by a Shimadzu UV-1800 spectrometer (Shimadzu Inc., Kyoto, Japan) at $270 \mathrm{~nm}$ using UV system ver.3.2 software (Toyama Sangyo Co., Osaka, Japan). It was made clear that none of the ingredients used in the formulations interfered with the assay. The release studies were conducted in triplicate, and the mean values were plotted versus time.

\section{Results and Discussion}

\subsection{Plasma treatment of HPMCAS powder}

We have already undertaken plasma irradiations on several cellulose derivatives such as EC, HEC, HPC, CMC, Chitin and Chitosan, and the radicals formed were studied by ESR with the aid of systematic computer simulations [8-10]. Based on a series of such studies, it was found that the surface of plasma-irradiated cellulose derivatives can be consisted of dangling-bond sites as a major component radical, indicative of the concurrent occurrence of the cross-link reaction in addition to a degradation reaction of cellulose main chain initiated by the 1,4glucoside bond scission. Likewise, HPMCAS, including highly plasma-crosslinkable side chain contained as a major substituent, can be assumed to form a cross-linked layer on the surface by plasma irradiation.

To determine the morphology changes induced by the plasma treatment, the surface of nontreated and treated HPMCAS powder were analyzed with SEM. It can be seen clearly from Fig. 2(a) that the particle size distribution of original HPMCAS powders is uniform in the size range of $2-5 \mu \mathrm{m}$. In the case of plasma treated samples, several agglomerates can be observed and also their average size was determined to be 20-70 $\mu \mathrm{m}$ (Fig. 2(b)).

\subsection{Degradation Property of HPMCAS by Plasma-Irradiation}

The degradation property of materials by plasma irradiation is known to be dependent on the plasma reaction apparatus and shape of the samples. In order to understand the nature of plasma-irradiation effect on degradation of nontreated and treated HPMCAS, we therefore studied the plasma degradation property of samples focusing on the changes in the weight 
loss of the directly-compressed tablets by plasma irradiation. Figure 3 illustrates changes in the weight loss according to the oxygen plasma irradiation time in comparison with the results obtained with typical cross-linkable PSt and degradable POM and lactose. (a)
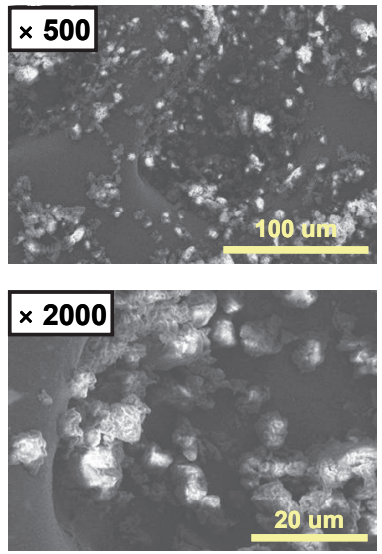

(b)
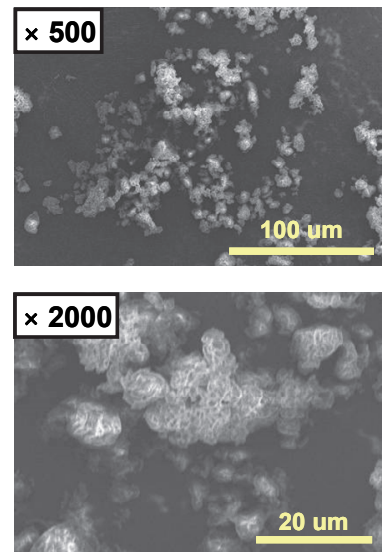

Fig. 2. SEM images of HPMCAS powders (a) before and (b) after plasma-irradiation.

Plasma condition: $50 \mathrm{~W}$, Ar $50 \mathrm{~mL} / \mathrm{min}, 66.6 \mathrm{~Pa}$.

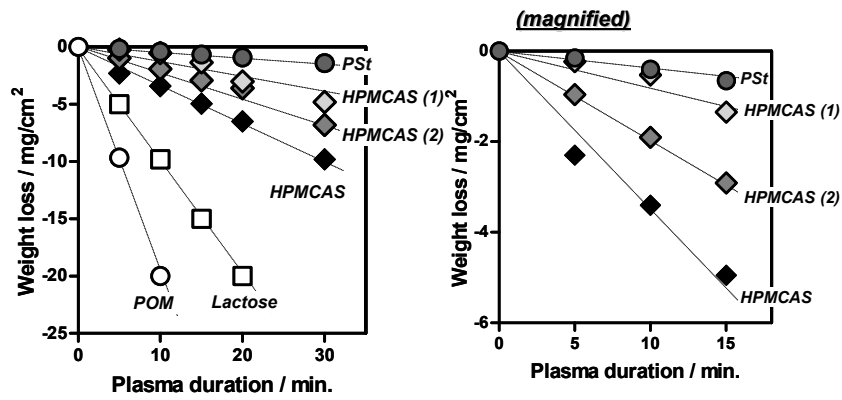

Fig. 3. Effect of oxygen plasma-irradiation on the degradation of flat-faced compressed tablets consisted of various compounds.

HPMCAS(1): Ar plasma pretreatment (10 W, $10 \mathrm{~min})$ HPMCAS(2): Ar plasma pretreatment $(20 \mathrm{~W}, 10 \mathrm{~min})$ Plasma condition: $50 \mathrm{~W}, \mathrm{O}_{2} 50 \mathrm{~mL} / \mathrm{min}, 66.6 \mathrm{~Pa}$.

It is seen that degradation rate of the treated HPMCAS are all lower than that of non-treated sample. This indicates that the cross-linked layer formation on the surface of HPMCAS powder by argon plasma pretreatment is effective to protect against oxidative decomposition with oxygen plasma irradiation.

Based on the fact that the value of weight loss increases as the plasma duration increases, it seems apparent that plasma degradable lactose could be selectively eliminated to result in the formation of the porous outer layer of the tablet. Then, drug could be released from the tablet through the resulting micropores.
3.3 Diclofenac Release from Plasma-Irradiated Double-Compressed Tablets

The two types of double-compressed tablets containing diclofenac sodium as a core material $(50 \mathrm{mg}, 10 \mathrm{~mm}$ in diameter) and mixture of pretreated or non-pretreated HPMCAS and lactose in 9:1 by weight as a wall material (mixture $100 \mathrm{mg}, 13 \mathrm{~mm}$ in diameter) were prepared. Subsequently, dissolution tests of the oxygen plasma-irradiated and non-irradiated tablets were carried out using the rotating basket apparatus method. Figure 4 illustrates the dependence of the release property of drug in a buffer solution at $\mathrm{pH} 7.4$ from the tablets on the plasma irradiation time. As is clear in Fig. 4, oxygen plasma non-irradiated tablet (blank) showed quick disintegration and burst release of drug at the very beginning due to combined effects of water absorption and swelling by the formulation. On the other hand, all the tablets plasma-irradiated for more than $5 \mathrm{~min}$ have clearly shown no burst release due to conversion into a sustained-release tablet. Moreover, the tablets consisted of pretreated HPMCAS gave a slower and continuous release profile over $8 \mathrm{~h}$ and showed near zero-order kinetics until approximately $50-70 \%$ of the drug in the tablet was released.

Thus, when the combinations of pretreated HPMCAS-Lactose (9:1) are used as a wall material of the double-compressed tablet, the plasma-irradiated tablets provided long term and stable drug release. It could be considered that the present tablets are apparently applicable to controlled-release systems having a desired release kinetic pattern by optimizing the plasma operational conditions.

The surface characteristics of the tested tablets were monitored by SEM observations. Figure 5 presents several SEM images on the surface of double-compressed tablets of pretreated- or nonpretreated-HPMCAS-Lactose, before and after plasma-irradiation for $10 \mathrm{~min}$. In comparison with surface of the blank tablet, the plasma-irradiated tablets have taken on very rough structure.

It is apparent that plasma irradiation caused cross-link reaction of HPMCAS, resulting in the formation of a non-uniform crosslinked barrier. Furthermore it is clearly seen that plasmairradiation generate a number of micropores due to the oxidative decomposition and/or vaporization of lactose monohydrate, resulting in 
the formation of the porous outer layer. It was also shown that the formation of fine-micropores with an approximate size of $2-10 \mu \mathrm{m}$ can be successfully achieved on the tablet consisted of argon plasma pretreated HPMCAS, as shown in

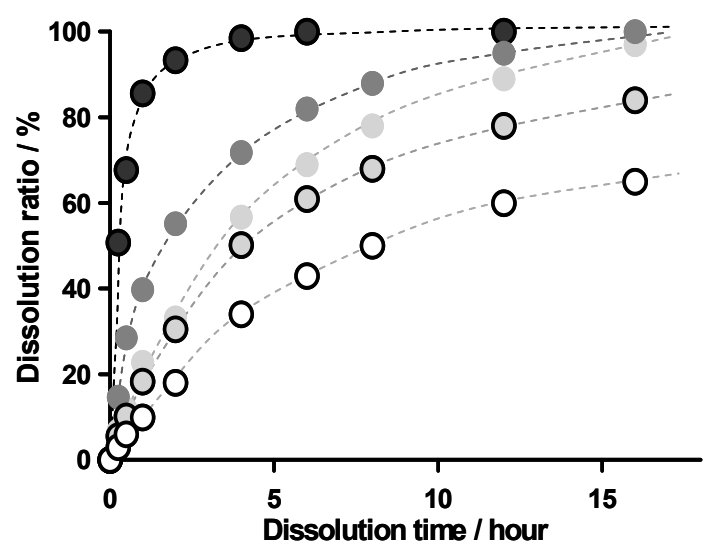

Fig 4. Effect of plasma durations on diclofenac release properties in $\mathrm{pH} 7.4$ buffer solution from plasma-irradiated double-compressed tablets using powdered mixtures of HPMCAS-Lactose $(9: 1)$ as outer layer.

Plasma conditions: $50 \mathrm{~W}, \mathrm{O}_{2} 50 \mathrm{~mL} / \mathrm{min}, 66.6 \mathrm{~Pa}$.

, blank;, 5 min duration (non-pretreatment); - $10 \mathrm{~min}$ duration (non-pretreatment); $0,10 \mathrm{~min}$ duration (Ar plasma pretreatment: $10 \mathrm{~W}, 10 \mathrm{~min}$ ); O, 10 min duration (Ar plasma pretreatment: $20 \mathrm{~W}, 10$ $\min )$. (a)

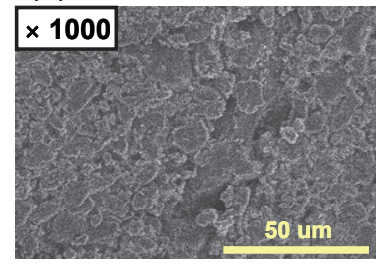

(b)

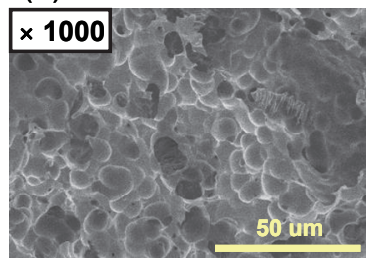

(c)

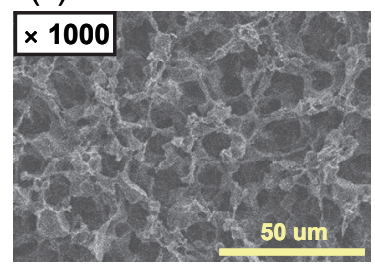

(d)

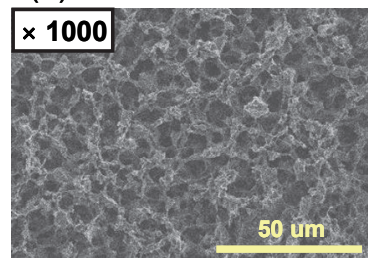

Fig. 5. SEM images of double-compressed tablets before and after plasma-irradiation for various duration.

Plasma conditions: $\mathrm{O}_{2} 50 \mathrm{~mL} / \mathrm{min}, 66.6 \mathrm{~Pa}$.

(a) blank; (b) 10 min duration (non-pretreatment);

(c) 10 min duration (Ar plasma pretreatment: $10 \mathrm{~W}, 10$ min); (d) 10 min duration (Ar plasma pre-treatment: $20 \mathrm{~W}, 10 \mathrm{~min})$.
Fig 5(d). This should be attributed to the protective effect of the pre-crosslinked layer against the oxidative decomposition.

\section{Conclusion}

We have developed a sustained release drug delivery system by formation of a number of fine-micropores with pore sizes mostly ranging from 2 to $10 \mu \mathrm{m}$ on the outer layer of two-layer tablet with plasma technique. The drug release profile from this system was confirmed in in vitro dissolution experiments. From the beginning of the dissolution test, a uniform release of drug was shown for more than 8 hours without spontaneous disintegration, and sustained release followed during 24-hours period. The results suggest that this system with plasma technique could be useful tool for the formulation strategy to obtain the desirable modulation of accurate drug release kinetics profile, according to its pharmacokinetics and therapeutic needs.

\section{References}

1. M. J. Gwen, R. R. Joseph, In G. S. Banker, C. T. Rhodes, Eds., Modern Pharmaceutics, $3^{\text {rd }}$ Ed., Vol. 72, Marcel Dekker Inc. New York, 1996, 575.

2. Y. W. Chein, Novel drug delivery systems. $2^{\text {nd }}$ ed., New York: Marcel Dekker Inc., 1997; pp.1-42.

3. M. Kuzuya, Y. Sasai, S. Kondo, Y. Yamauchi, Curr. Drug Discov. Tech., 6 (2009) 135.

4. M. Kuzuya, Y. Sasai, Y. Yamauchi, S. Kondo, Industrial Plasma Technology: Applications from Environmental to Energy Technologies, WILEY- VCH Verlag GmbH \& Co,in part, (2010) pp.113.

5. Y. Sasai, S. Kondo, Y. Yamauchi, M. Kuzuya, Biomecdical Engineering, INTECH, (2011) pp101.

6. M. Kuzuya, A. Noguchi, H. Ito, M. Ishikawa, Drug Delivery System, 6 (1991) 119.

7. Y. Yamauchi, M. Kuzuya, Y. Sasai, S. Kondo, J. Photopolym. Sci. Technol., 25 (2012) 535.

8. M. Kuzuya, Y. Yamauchi, J. Niwa, S. Kondo, Y. Sakai, Chem. Pharm. Bull., 43 (1995) 2037.

9. M. Kuzuya, Y. Yamauchi, J. Niwa, S. Kondo, Proc. SPSM, 9 (1996) 55.

10. M. Kuzuya, Y. Sasai, S. Kondo, J. Photopolym. Sci. Tecnol., 12 (1999) 75. 